\title{
Evaluation of bioluminescence-based assays of anti-malarial drug activity
}

\author{
Sandra Hasenkamp ${ }^{1}$, Adam Sidaway ${ }^{2}$, Oliver Devine ${ }^{3}$, Richard Roye ${ }^{2}$ and Paul Horrocks ${ }^{1,3^{*}}$
}

\begin{abstract}
Background: Transgenic Plasmodium falciparum expressing luciferase offers an attractive bioluminescence-based assay platform for the investigation of the pharmacological properties of anti-malarial drugs. Here a side-by-side comparison of bioluminescence and fluorescence-based assays, utilizing a luciferase reporter cassette that confers a strong temporal pattern of luciferase expression during the S-phase of intraerythrocytic development, is reported.

Methods: Key assay parameters for a range of commercially available luminogenic substrates are determined and compared to those measured using a Malaria Sybr Green I fluorescence assay. In addition, the short-term temporal effects of anti-malarial compounds are evaluated using both bioluminescent and fluorescent assay platforms.

Results: The Z', \% coefficient of variation and 50\% inhibition concentrations are essentially the same for bioluminescent and fluorescent assays in transgenic parasites generated in both chloroquine-sensitive and -resistant genetic backgrounds. Bioluminescent assays, irrespective of the luminogenic agent employed, do, however, offer significantly enhanced signal-to-noise ratios. Moreover, the bioluminescent assay is more dynamic in terms of determining temporal effects immediately following drug perturbation.

Conclusion: This study suggests that opportunities for bioluminescence-based assays lie not in the measurement of 50\% inhibition concentrations, where the cheaper fluorescence assay performs excellently and is not restricted by the need to genetically modify the parasite clone under investigation. Instead, assays that use the dynamic response of the luciferase reporter for semi-automated screening of additional pharmacological properties, such as relative rate-of-kill and lethal dose estimation, are a more attractive development opportunity.
\end{abstract}

Keywords: Luciferase, Sybr green I, Antimalarial drug, Relative rate of kill, Malaria

\section{Background}

Following the emergence and spread of multidrug resistant Plasmodium falciparum and reports of artemisinin treatment failure in South-East Asia, there is an urgent need to identify new small molecule drugs for the treatment of malaria [1,2]. Towards this aim, several recent reports have described the high throughput screening (HTS) of massive chemical libraries and the identification of novel chemical scaffolds to be taken forward for development [3-5]. Prioritizing these compounds for further exploration of their pharmacokinetic and pharmacodynamic properties is essential and has been the focus of several recent developments in assay formats [6-10].

\footnotetext{
* Correspondence: p.d.horrocks@keele.ac.uk

'Institute for Science and Technology in Medicine, Keele University, Staffordshire, UK

${ }^{3}$ School of Medicine, Keele University, Staffordshire, ST5 5BG, UK

Full list of author information is available at the end of the article
}

Assays of anti-malarial drug activity typically rely on either radiometric or fluorescence-based platforms. Incorporation of tritiated hypoxanthine or ethanolamine has been widely used as a biomarker for growth and offers a sensitive assay with excellent signal/noise $(\mathrm{S} / \mathrm{N})$ ratio [11]. There are, however, significant issues around the safe storage and disposal of the radiolabelled material. Fluorescence-based platforms, such as the use of the DNA intercalating agents Sybr Green I or 4',6'-diamidino-2-phenylindole (DAPI), offer a rapid, cheap and sensitive assay but can be problematic due to the low $\mathrm{S} /$ $\mathrm{N}$ ratio resulting from high background signals [12-14]. That said, the utility of the Malaria Sybr Green I Fluorescence (MSF) assay for single-dose HTS has been readily demonstrated.

A recent innovation has been the adoption of a bioluminescence assay platform that utilizes transgenic

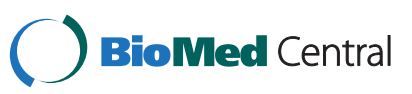

(c) 2013 Hasenkamp et al.; licensee BioMed Central Ltd. This is an Open Access article distributed under the terms of the Creative Commons Attribution License (http://creativecommons.org/licenses/by/2.0), which permits unrestricted use, distribution, and reproduction in any medium, provided the original work is properly cited. 
P. falciparum expressing the luciferase reporter gene $(l u c)$. The utility of bioluminescence has been demonstrated in HTS of medium-sized chemical libraries, exploration of gametocidal and delayed-death drug action, and the development of a murine malaria assay in Plasmodium berghei [15-20]. The use of bioluminescence to assay anti-malarial drug activity in P. falciparum was first described in 2008 [17]. Here luc was flanked by the Pfhsp $865^{\prime}$ and Pbdhfr-ts $3^{\prime}$ sequences and integrated as a short concatamer into chromosome 7 . The assay provided $50 \%$ inhibition concentration $\left(\mathrm{IC}_{50}\right)$ results comparable to those determined using ${ }^{3} \mathrm{H}$-hypoxanthine or MSF, with a subsequent report demonstrating the feasibility of scaling the use of this transgenic parasite into a 384-multiwell format [16]. Screening the Library of Pharmacologically Active Compounds (LOPAC ${ }^{1280}$ ) demonstrated the robustness of this assay format with $\mathrm{Z}^{\prime}$ scores $>0.7$ and $\mathrm{S} / \mathrm{N}$ ratio of 71. In 2010, a second luc transgenic parasite with a Pfhrp 2 promoter was reported for the screening of three chemical libraries, a total of 12,320 compounds, reporting Z' scores of 0.64-0.76 and S/N ratios of 147-430 [20]. The increase in $\mathrm{S} / \mathrm{N}$ ratio recorded here presumably represents their choice of the Bright-Glo luminogenic substrate in their assays. A final report describes the $b x b \mathrm{I}$ integrasemediated integration of a Pfhrp3-luc-Pfhrp2 cassette into chloroquine-resistant (CQR) and -sensitive (CQS) genetic backgrounds [18]. Whilst the bioluminescence assay data correlated well with that generated using ${ }^{3} \mathrm{H}$-hypoxanthine and MSF for fast-acting drugs (Z' score of 0.71 and $\mathrm{S} / \mathrm{N}$ of 51 ), the MSF assay appeared to provide poor resolving capacity when screening delayed-action drugs such as azithromycin ( $Z^{\prime}$ scores $<0.4$ and $\mathrm{S} / \mathrm{N}$ of $2-3$ ).

The generation of a transgenic parasite where luc is under the control of the $5^{\prime}$ and $3^{\prime}$ flanking sequences of Pfpcna has previously been described $[21,22]$. These flanking sequences have been shown to provide the regulatory elements necessary to reconstitute the absolute and temporal control of the endogenous Pfpcna gene on the luc reporter gene. Pfpcna encodes a processivity factor for DNA polymerase $\delta$ and, as such, acts as a biomarker for DNA replication in mature trophozoites during intraerythrocytic schizogony [23]. This transgenic parasite shows a strong 20-50,000 fold-increase in luciferase signal in mature trophozoites when compared to ring stage parasites, suggesting that the matched Pfpcna $5^{\prime}$ and $3^{\prime}$ flanking sequences confer a strong dynamic temporal control on luc [22]. Here an evaluation of the Pfpcna-luc transgenic parasite as a tool to explore the action of anti-malarial drugs is described along with a side-by-side comparison of this assay with MSF in both CQS and CQR genetic backgrounds. This study offers insights into the opportunities available for bioluminescence assays to explore a range of pharmacological properties of anti-malarial drugs in a format readily scalable for high throughput assays.

\section{Methods}

\section{Plasmodium falciparum cell culture}

The transgenic Dd2 P. falciparum clone expressing luciferase under the control of Pfpcna flanking sequences $\left(\mathrm{Dd} 2^{l u c}\right)$ has been previously described [21]. $\mathrm{Dd} 2^{\text {luc }}$ were cultured using standard continuous culture conditions (RPMI640 medium supplemented with 37.5 mM HEPES, $10 \mathrm{mM}$ D-glucose, $2 \mathrm{mM}$ L-glutamine, $100 \mu \mathrm{M}$ hypoxanthine, $25 \mu \mathrm{gml}^{-1}$ gentamycin and $8 \% \mathrm{v} / \mathrm{v}$ human serum) at a $2 \%$ haematocrit in an atmosphere of $1 \% \mathrm{O}_{2}, 3 \% \mathrm{CO}_{2}$, and $96 \% \mathrm{~N}_{2}$. 5nM WR99210 and $2.5 \mu \mathrm{g} / \mathrm{ml}$ blasticidin S drug selection was applied throughout. Staging and parasitaemia were determined by light microscopy of Giemsastained thin blood smears. Synchronization of cultures was attained using sequential sorbitol lysis treatment [24].

\section{Generation of NF54 ${ }^{\text {luc }}$ transgenic parasite}

NF54 ${ }^{a t t B}$ and pINTNeo were kindly provided by David Fidock (Columbia University, New York). BxbI integrase-mediated integration of the same Pfpcna-luc reporter construct present in $\mathrm{Dd} 2^{l u c}$ was performed in NF54 ${ }^{a t t B}$ as previously described, except that WR99210 selection was not applied as the hdhfr selection cassette is omitted from NF54 ${ }^{a t t B}[21,25]$. Integration was confirmed by absence of PCR over the cg6 locus, in which the $a t t B$ site is located (primers P1, 5'ATGAACAAA TACATAAGAGCGC3' and P2, 5'TCTTTAATTTTA TTTTGGTCATGC3'), and generation of a PCR product over the attL site (primers P1 and P3, 5'TAAGGAGAA AATACCGCATCAGG3') that results from attP $\times$ attB recombination.

The reconstituted temporal control of Pfpcna expression over luc was shown by stage-specific northern blot and luciferase assay. NF54 $4^{\text {luc }}$ was synchronized and samples removed at five points during asexual intraerythrocytic development. These samples represented major stages of morphological development as judged by light microscopy; early rings (0-8 hours post-infection, hpi), late rings (9-16hpi), early trophozoites (17-24hpi), late trophozoites (25-36hpi) and schizonts (37-48hpi), termed $\mathrm{T} 1$ to $\mathrm{T} 5$, respectively. Luciferase assays on $\mathrm{T} 1$ to T5 were carried out as described below. For stage-specific northern blots, total RNA was isolated from samples $\mathrm{T} 1$ to $\mathrm{T} 5$ and $5 \mu \mathrm{g}$ of total RNA was size-fractionated, blotted and hybridized to $\alpha-{ }^{32} \mathrm{P}$ dATP random-primer labelled probes to the $l u c$ and Pfpcna genes. Images were captured using the Cyclone Storage Phosphor screen apparatus (Packard) and analysed using OptiQuant software (Packard).

\section{Standard luciferase assay procedure and time course}

A standard single-step lysis procedure was used throughout [22]. Forty $\mu \mathrm{l}$ samples of $P$. falciparum culture (typically at $2 \%$ haematocrit, $\mathrm{HCT}$ ) were transferred to a well 
on a white 96-multiwell plate (Greiner, UK) and $10 \mu \mathrm{l}$ of passive lysis buffer (Promega, UK) added and homogenized by shaking the plate. An equal volume, $50 \mu \mathrm{l}$, of luminogenic substrate was mixed with the lysed parasites and the bioluminescence (in relative light units, RLU) was measured for $2 \mathrm{sec}$ in a Glomax Multi Detection System (Promega, UK). Four commercially available (Promega, UK) luminogenic substrates were used in this study: standard luciferase (SL) substrate, Bright-Glo ${ }^{\mathrm{TM}}$, One-Glo ${ }^{\mathrm{TM}}$ and Steady-Glo ${ }^{\mathrm{TM}}$.

For the timecourse assay, a $2 \%$ trophozoite-stage Dd $2^{\text {luc }}$ culture at $2 \% \mathrm{HCT}$ was prepared. Five $40 \mu \mathrm{l}$ samples were tested with each luminogenic reagent and the bioluminescent signal from each sample monitored over 100 mins immediately thereafter. Mean RLU \pm standard deviation (stdev) were plotted against time. To determine the relative decay of samples, the RLU measured at each timepoint was expressed as a fraction of the initial mean RLU with the mean fraction \pm stdev plotted against time.

\section{Dynamics of bioluminescence and fluorescence following drug treatment}

A $2 \%$ early trophozoite-stage (17-24hpi) Dd2 ${ }^{\text {luc }}$ culture at $2 \% \mathrm{HCT}$ was prepared and divided into $2 \mathrm{ml}$ aliquots; one for each drug/assay to be tested and a further nodrug control. At $t=0$ hrs, either three $40 \mu \mathrm{l}$ aliquots were removed to determine the initial bioluminescent signal or three $100 \mu \mathrm{l}$ samples removed to measure the fluorescent signal (see below). The drug being tested was added at this time. At the timepoint indicated, samples of the appropriate volume were removed and the bioluminescent/fluorescent signal measured. The mean \pm stdev fraction of the bioluminescent/fluorescent signal measured from the drug-treated samples was normalized against the mean bioluminescent/fluorescent signal from the no-drug control at the same timepoint.

Bioluminescent signals were measured using the protocol described above with the SL substrate. Fluorescent signals were measured using a standard MSF assay [14]. Specifically, an equal volume of MSF lysis buffer $(100 \mu \mathrm{l}$ of $20 \mathrm{mM}$ Tris (pH 7.5), $5 \mathrm{mM}$ EDTA, 0.008\% (w/v) saponin and $0.08 \%(\mathrm{v} / \mathrm{v})$ Triton $\mathrm{X}-100)$ containing SYBR green I $(1 \times$ final concentration, from $5000 x$ stock supplied by Invitrogen, UK) was added to $100 \mu \mathrm{l}$ of $\mathrm{Dd} 2^{l u c}$ aliquoted onto a black 96-multiwell plate (Greiner, UK). Well contents were homogenized by repeated pipetting and incubated for one hour in the dark at room temperature. The fluorescent signal, in RLU, was measured using the blue fluorescent module (excitation $490 \mathrm{~nm}$ : emission 510-570 $\mathrm{nm}$ ) of a Glomax Multi Detection System (Promega, UK).

Measuring $I C_{50}$ using luciferase and MSF assay formats Trophozoite-stage cultures of the transgenic line being tested (100 $\mu \mathrm{l}, 2 \%$ parasitaemia, 4\% HCT, $\mathrm{n}=3)$ were added to 96-multiwell plates containing $100 \mu \mathrm{l}$ of predosed (five-fold dilution series) complete medium. On each assay plate, six wells containing $200 \mu \mathrm{l}$ of $2 \%$ parasitaemia cell culture $(2 \% \mathrm{HCT})$ in the absence of drugs served as a positive control (100\%), whereas the same culture mix in the presence of a $1 \mu \mathrm{M}$ supralethal dose of artemether served as a negative growth control (0\%). The outermost wells on each plate contained $200 \mu \mathrm{l}$ of incomplete medium to minimize edge effects from evaporation during $48 \mathrm{hr}$ incubation in a gassed $\left(1 \% \mathrm{O}_{2}, 3 \% \mathrm{CO}_{2}\right.$, and $96 \% \mathrm{~N}_{2}$ ) chamber at $37^{\circ} \mathrm{C}$.

For bioluminescent assays, RLU were measured using the SL substrate. Irrespective of the assay, the \% growth was calculated as follows: $100 x\left[\mu_{(S)}-\mu_{(-)} / \mu_{(+)}-\mu_{(-)}\right]$ where $\mu_{(S)}, \mu_{(+)}$and $\mu_{(-)}$represent the means for the sample in question and $100 \%$ and $0 \%$ controls, respectively. The $\%$ growth was plotted against $\log _{10}$-transformed drug concentration and the $\mathrm{IC}_{50}$ determined using a nonlinear regression (sigmoidal dose-response/variable slope equation) in GraphPad Prism v5.0 (GraphPad Software, Inc., San Diego, CA).

\section{Determination of assay quality parameters}

Derivation of the statistical tests of the assay quality parameters; $\mathrm{Z}$ ' score, $\% \mathrm{CV}_{\max }, \% \mathrm{CV}_{\text {min }}$ and signal/noise ratio was as described [26]. The $Z^{\prime}$ score was calculated : $Z^{\prime}=1-\left[\left(3 \sigma_{(+)}+3 \sigma_{(-)}\right) / \mu_{(+)}-\mu_{(-)}\right]$, where $\mu_{(+)}$and $\sigma_{(+)}$ are the mean and stdev of the no-drug positive control, respectively; $\mu_{(-)}$and $\sigma_{(-)}$are the mean and stdev of a supralethal kill with $1 \mu \mathrm{M}$ of artemether (negative control), respectively.\% $\mathrm{CV}_{\max }$ was calculated: $100 x\left[\sigma_{(+)} / \mu_{(+)}\right]$ and $\% \mathrm{CV}_{\text {min }}: 100 \mathrm{x}\left[\sigma_{(-)} / \mu_{(-)}\right]$. The $\mathrm{S} / \mathrm{N}$ ratio was calculated: $\left[\mu_{(+)}-\mu_{(-)}\right] / \sigma_{(-)}$.

\section{Results}

\section{Evaluating the parameters of the Pfpcna-luc} bioluminescence assay

The generation of the Pfpcna-luc transgenic parasite in the $\mathrm{Dd} 2(\mathrm{CQR})$ genetic background $\left(\mathrm{Dd} 2^{l u c}\right.$ ) has been previously described [21]. The haematocrit and parasitaemia starting conditions suitable for a 96-multiwell format assay using trophozoite stage $\mathrm{Dd} 2^{\text {luc }}$ were established using a range of parasitaemia $(0.5-5 \%)$ at three different haematocrit (HCT; 1,2 and 4\%). The standard luciferase (SL) luminogenic substate was used to measure bioluminescence in relative light units (RLU). Correlating RLU against parasitaemia for each HCT used (Figure 1) shows a strong linear relationship (all $\mathrm{R}^{2}>$ 0.97) irrespective of the HCT. Increasing the parasite number through fold-increases in $\mathrm{HCT}$ does initially (1 to $2 \% \mathrm{HCT}$ ) result in similar fold-increases in RLU; however, the fold-increase in RLU between $2 \%$ and $4 \%$ HCT is markedly reduced due to the quenching effect of the additional haemoglobin released at these higher 


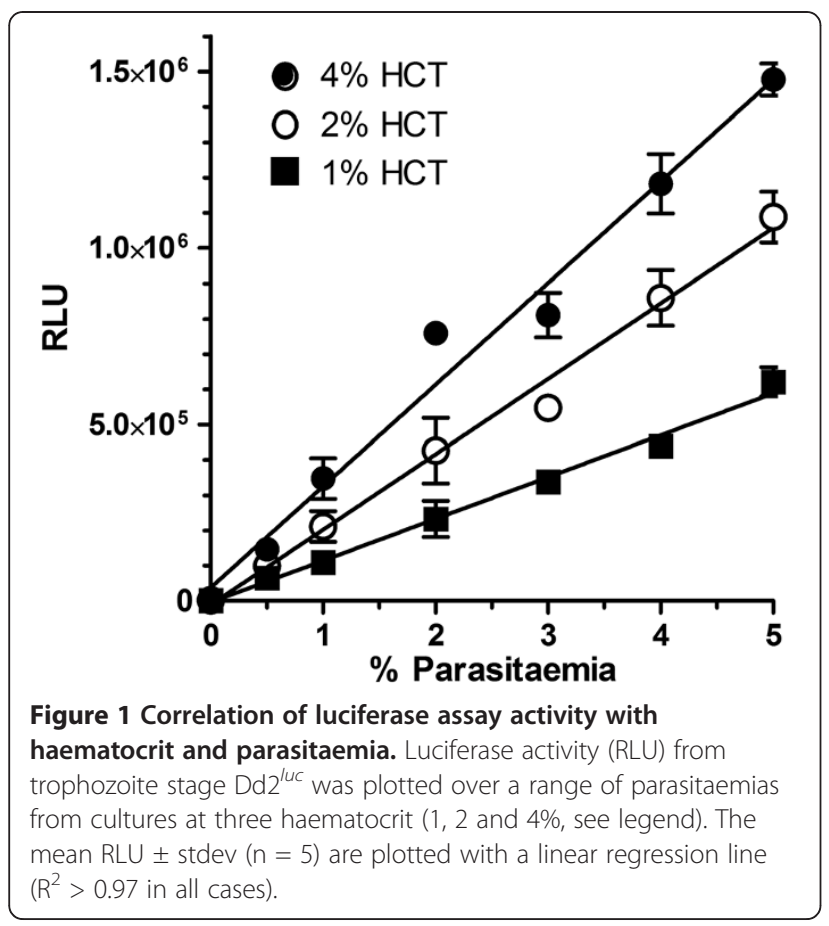

HCT [16,22]. Starting conditions of $2 \%$ HCT and $2 \%$ trophozoite-stage parasitaemia were selected for all future experiments. The selection of $2 \%$ parasitaemia balances the effect of kill ( $2 \%$ and below) and growth (assuming a 2-3 fold increase in parasitaemia) on the linear response curve.

The absolute signal intensity and time-dependent decay of RLU generated from a range of commercially available luminogenic substrates was next explored. The luminogenic substrates were added to lysed $\mathrm{Dd} 2^{l u c}$ and the mean RLU ( $(n=5)$ measured over 100 minutes (Figure 2A). The
Bright-Glo substrate provided the highest initial absolute signal, some $60-80 \%$ higher than those for the SL and One-Glo substrates and 20-fold higher than that of Steady-Glo. Following the time-dependent decay of each luminogenic substrate, determined in terms of absolute RLU (Figure 2A) or as a fraction of initial RLU (Figure 2B), shows that the signal from the Steady-Glo substrate remains essentially unchanged over the 100 minutes assayed, whilst those of Bright-Glo, SL and One-Glo substrates decay to $10 \%, 22 \%$ and $28 \%$ of the original signal, respectively. Importantly, within the first five minutes, a time in which a 96-multiwell plate can be readily measured, there is a maximum loss of $10 \%$ of the initial RLU irrespective of the luminogenic substrate chosen.

The effect of choice of luminogenic substrate for the determination of $\mathrm{IC}_{50}$ values was evaluated using chloroquine (CQ) and artemether (ART). Comparison of the absolute RLU (Figures 3A and 3C) over the range of drug concentrations tested shows the expected relative relationship. Normalizing the RLU as a fraction of the untreated control for each luminogenic substrate generates overlapping non-linear sigmoidal dose-response curves that provide essentially identical $\mathrm{IC}_{50}$ values irrespective of the substrate chosen (Figures $3 \mathrm{~B}$ and 3D). Ten samples of Dd2 ${ }^{l u c}$ exposed for 48 hrs to either no drug (100\% growth) or a supralethal dose of ART $(1 \mu \mathrm{M}$, $0 \%$ growth) allow the $\mathrm{Z}^{\prime}$ score, $\mathrm{S} / \mathrm{N}$ ratio and coefficient of variation $(\% \mathrm{CV})$ assay parameters to be determined for each luminogenic substrate in a 96-multiwell format (Table 1). For comparison, the same assay parameters were determined using the MSF assay (Table 1). These data indicate that whilst the $Z^{\prime}$ scores are essentially the same for all the assay formats tested, and all formats would be readily suitable for HTS, there were significant
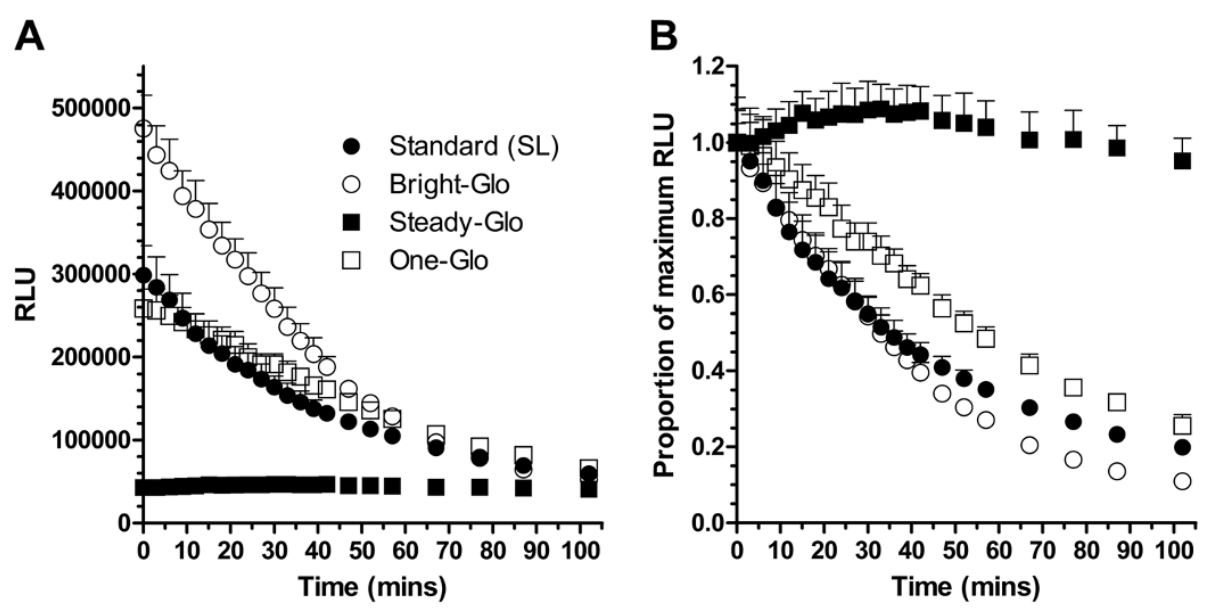

Figure 2 Absolute and time-dependent decay of luminogenic substrates. Trophozoite stage Dd2luc (2\% parasitaemia, $2 \%$ HCT) were lysed and an equal volume of the indicated (see key) luminogenic substrate added. (A) The decay in absolute mean value of RLU $\pm s t d e v(n=5)$ for each luminogenic substrate (see key) is plotted over 100 minutes. (B) The relative decay for each luminogenic substrate is reported by plotting the mean RLU \pm stdev as a proportion of the mean RLU at $\mathrm{t}=0$ mins. 

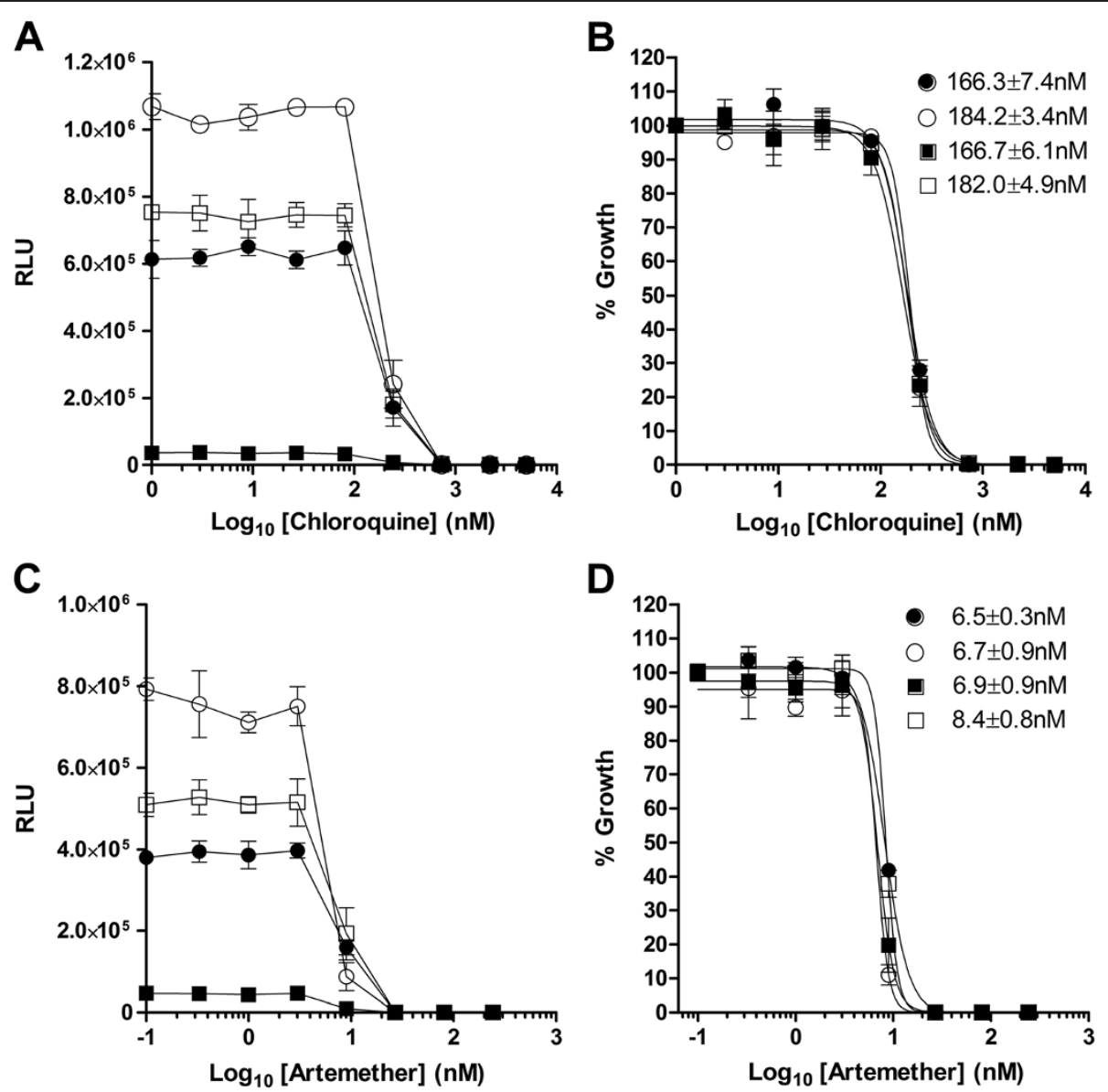

Figure 3 The effect of luminogenic substrate choice on the determination of $\mathbf{I} \mathbf{C}_{50}$ values. Trophozoite stage Dd2 ${ }^{\text {luc }}(2 \%$ parasitaemia, $2 \% \mathrm{HCT}$ ) were incubated for $48 \mathrm{hrs}$ in the presence of the indicated concentration of drug prior to measurement of the absolute mean $\mathrm{RLU} \pm \operatorname{stdev}(\mathrm{n}=3)$ using $(\mathbf{A})$ chloroquine and $(\mathbf{C})$ artemether. Normalizing these data as a fraction of the controls (\% growth) allows a dose-response curve to be plotted for each luminogenic substrate for $(\mathbf{B})$ chloroquine and (D) artemether. The $I_{50}$ values determined for each luminogenic substrate (see key in Figure 2) are shown.

differences in the $\mathrm{S} / \mathrm{N}$ ratios. Critically, luminogenic substrate provides $\mathrm{S} / \mathrm{N}$ ratios between 469 and 4042 fold higher than those of the fluorescence-based MSF assay. As all the luminogenic substrates share a similar and very low background signal (50-80 RLU), the fold increase in $\mathrm{S} / \mathrm{N}$ ratio correlates with the initial absolute RLU generated by each substrate. The low variation in the maximal signal ( $100 \%$ growth, $\left.\% \mathrm{CV}_{\max }\right)$ across

Table 1 Comparison of assay parameters from bioluminescence and fluorescence-based assays

\begin{tabular}{lcccc}
\hline Assay reagent & Z'score $^{\prime}$ & Signal/noise ratio & \%CV $_{\max }$ & \%CV $_{\mathbf{m i n}}$ \\
\hline Bright-Glo & $0.74-0.84$ & $15667-17746$ & $5.2-6.7$ & $14.8-16.2$ \\
SL substrate $^{1}$ & $0.73-0.81$ & $12853-13609$ & $8.0-8.9$ & $15.5-17.0$ \\
One-Glo & $0.77-0.86$ & $8936-10030$ & $4.7-6.3$ & $14.3-19.5$ \\
Steady-Glo & $0.83-0.86$ & $2063-2344$ & $5.6-7.1$ & $17.2-18.6$ \\
MSF & $0.72-0.79$ & $4.30-4.39$ & $4.0-6.6$ & $2.8-5.8$ \\
\hline
\end{tabular}

${ }^{1}$ Standard luciferase (SL). all the luminogenic substrates demonstrates the robust performance of the bioluminescence assays in generating consistent data. The higher $\% \mathrm{CV}_{\min }$ provided by the bioluminescence assays is a direct result of variation of the very low background signal (50-80 RLU).

Bioluminescence is a more dynamic reporter of drug action The fluorescence and bioluminescence assays of $\operatorname{Dd} 2^{l u c}$ both monitor DNA replication. MSF directly monitors DNA content and bioluminescence measures the S-phase-linked induction of expression of DNA replication proteins. However, the DNA biomarker is much more stable than the luciferase biomarker, and each is subject to distinct cellular regulation processes. To explore the effect of the stability of these different biomarkers, a lethal drug perturbation was induced and the immediate dynamic responses of the bioluminescence and fluorescent assays followed. For the bioluminescence assays, the SL substrate 
was selected for use throughout, its choice reflecting a balance of signal intensity, stability and relative cost.

$\operatorname{Dd} 2^{\text {luc }}$ was exposed to supralethal doses of drug perturbation; kill was induced using $1 \mu \mathrm{M}$ of the RNA polymerase inhibitor Actinomycin D (ActD) or the ribosome translational elongation inhibitor cyclohexamide (CHX). Over 8 hrs, samples were harvested and the bioluminescent/fluorescent signal measured. In each case, the RLU measured were expressed as a fraction of the mean RLU from an untreated control harvested at the same timepoint to compensate for the normal temporal programme of $l u c$ expression over the course of the experiment.

Kill induced by either drug results in decreasing bioluminescent/fluorescent signal over the 8 hours assayed (Figure 4A). Whereas the bioluminescent signal decreased to between 3 and $22 \%$ of the untreated control (square symbols in Figure 4A), the fluorescent signal only decreased to between 57 and $65 \%$ of the untreated control over the same period (circles in Figure 4A). Interestingly, the fluorescent signal initially decreased more on ActD treatment, whereas the bioluminescent signal was most affected by CHX. These data suggest that the action of ActD appears to initially reduce Sybr Green I binding, presumably through low-affinity binding to a component of double-stranded RNA, with the inhibition of de novo DNA synthesis by CHX slightly delayed. The residual $65 \%$ of fluorescence even after 8 hours of supralethal drug treatment presumably reflects the stability of the nucleic acid biomarker even after cell death. By contrast, supralethal doses of $\mathrm{CHX}$ rapidly block the de novo synthesis of luciferase and the induced kill leads to a rapid decline in bioluminescence. Over the 8 hours of treatment, the bioluminescent signal decreases to $3 \%$ of the untreated control, with the timeresponse curve indicating that the luciferase protein has a half-life of approximately 1.5 hours in P. falciparum. The action of ActD appears slightly delayed, presumably as luc transcript is available for translation prior to cell death.

Based on the initial dynamic response of the bioluminescence assay to ActD and CHX, the effect of antimalarial drugs was similarly tested. Four drugs were selected; dihydroartemisinin (DHA, 20nM), artemether (ART, 25nM), chloroquine (CQ, 500nM) and azithromycin (AZ, $6 \mu \mathrm{M})$ with the concentration of each drug representing the $3 \mathrm{xIC}_{50}$ dose (see below). The timeresponse curves generated provide relative rate-of-kill data that follows the known relative action of these drugs (Figure 4B) [10]. The artemisinins ART and DHA provide the fastest rate of kill. CQ represents an intermediate between the artemisinins and AZ, a drug that shows a delayed-death phenotype. The killing-effect of AZ would not be expected to be exerted within the 8 hour time period used here, instead requiring the parasite to complete at least one full cycle of intraerythrocytic development [18].

\section{Side by side comparison of bioluminescence $\mathbf{v}$.} fluorescence determination of $\mathrm{IC}_{50}$

As luciferase expression in $\mathrm{Dd} 2^{l u c}$ is temporally linked to DNA replication, this parasite offers the most appropriate bioluminescence assay for a side by side comparison with the DNA intercalating action of Sybr Green I in the MSF assay. To extend the comparison of $\mathrm{IC}_{50}$ derived from bioluminescence and fluorescence assays using the Pfpcna-luc cassette, this cassette was also introduced
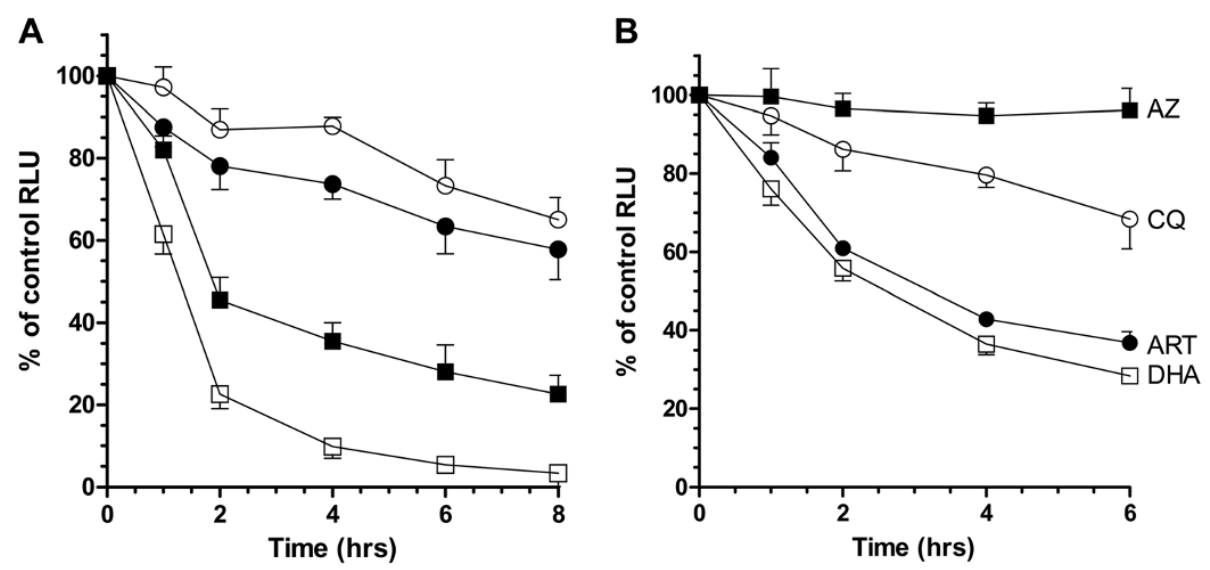

Figure 4 Exploring the immediate dynamic response of bioluminescence and fluorescence assays of drug activity. (A) Plot of the time-dependent changes in relative RLU (bioluminescent and fluorescence when compared to untreated control) measured from trophozoite stage Dd2 $2^{\text {luc }}$ (2\% parasitaemia, $2 \%$ HCT) exposed to a supralethal $1 \mu \mathrm{M}$ dose of actinomycin D (filled symbol) or cyclohexamide (open symbol). The fraction of mean RLU $\pm \operatorname{stdev}(n=3)$ at each timepoint are shown for a luciferase assay (square) or MSF assay (circle). (B) Plot of the time-dependent changes in mean fraction of luciferase RLU $\pm \operatorname{stdev}(n=3)$ exposed to $3 \times C_{50}$ doses of the indicated anti-malarial drug; artemether (ART), azithromycin (AZ), dihydroartemisinin (DHA) and chloroquine (CQ). 
into the CQS genetic background provided by NF54. The Pfpcna-luc cassette was introduced into NF54 ${ }^{\text {attB }}$ by $b x b \mathrm{I}$-mediated integration (Figure $5 \mathrm{~A}$ ) as confirmed by PCR analysis (Figure 5B). To demonstrate that the same temporal control of Pfpcna was reconstituted over the luc gene in NF54 ${ }^{a t t B}$, stage-specific northern blots and luciferase assays were performed (Figures $5 \mathrm{C}$ and $5 \mathrm{D}$ ). Taking five timepoints ( $\mathrm{T} 1$ to $\mathrm{T} 5$ ) that represent major morphological stages during asexual intraerythrocytic development (early ring, late ring, early trophozoite, late trophozoite and schizont, respectively), the expected patterns of temporal steady-state transcript levels and luciferase activity were demonstrated, with the highest levels for both coinciding with S-phase in late trophozoites (sample T4) [22].

Using the luciferase transgenic lines Dd2 ${ }^{l u c}$ and NF54 $4^{l u c}$, the $\mathrm{IC}_{50} \mathrm{~s}$ for eight anti-malarial drugs, representing a range of modes of action, were determined using both bioluminescence and MSF assays (Table 2). The parental lines $\mathrm{Dd} 2^{\text {attB }}$ and NF54 $4^{\text {attB }}$ were included using the MSF assay alone as a control to explore any effect on $\mathrm{IC}_{50}$ resulting from the genetic modification with the Pfpcna-luc cassette. Irrespective of the assay format, the $\mathrm{IC}_{50} \mathrm{~s}$ determined for these drugs were essentially the same. The differences in $\mathrm{IC}_{50}$ for $\mathrm{CQ}$ and quinine $(\mathrm{QN})$ between $\mathrm{Dd} 2^{l u c}$ and NF54 ${ }^{l u c}$ reflect known differences in their resistance profiles for these quinolone drugs. Determining the mean $\mathrm{IC}_{50}$ ratio for $\mathrm{Dd} 2^{\text {luc }} / \mathrm{NF} 54^{\text {luc }}$ for $\mathrm{CQ}$ gave values of 8.57 and 8.91 for the bioluminescent and MSF assays, respectively. Similarly, for QN, the ratios were almost identical; 2.38 and 2.28 , and reflect typical values for these drugs in CQR/CQS parasites. The $\mathrm{IC}_{50}$ values derived using the MSF assay for the docking parasite lines Dd2 $2^{\text {attB }}$ and NF54 $4^{\text {attB }}$ were similarly
A

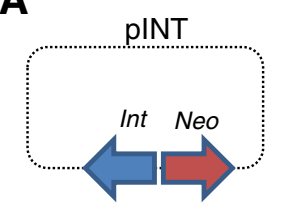

Int mediated recombination X
B
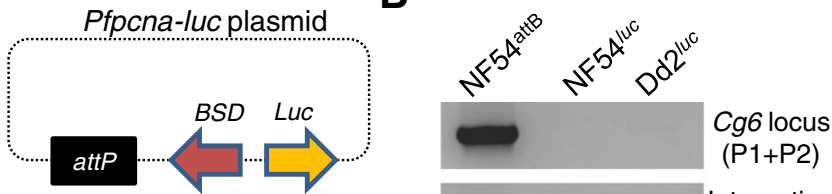

Integration (P1+P3)
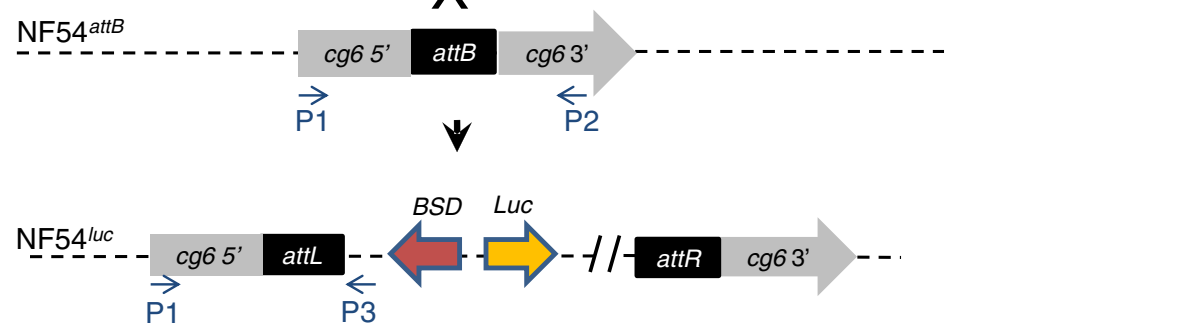

C
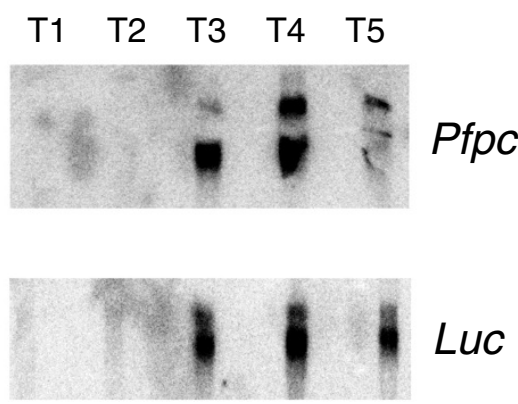

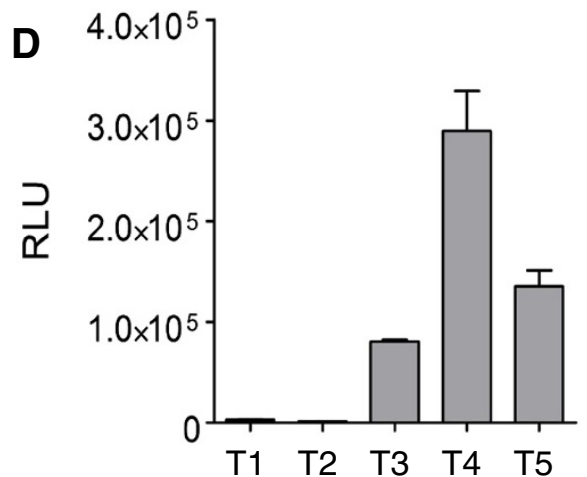

Figure 5 Generation of the NF54 ${ }^{\text {Iuc }}$ transgenic line. (A) Schematic representing the bxbl-integrase (Int) mediated integration of the Pfpcna-luc plasmid into the cg6-attB locus in NF54 ${ }^{a t t B}$. BSD and Neo represent the drug selection markers for blasticidin S and neomycin, respectively. The position and orientation of oligonucleotide primers (P1 to P3) used to confirm integration are shown. (B) PCR monitoring of integration of Pfpcna-luc plasmid. The top panel shows results of PCR across the cg6-attB locus (primers P1 and P2), and the lower panel, PCR across the attL junction (primers P1 and P3) resulting from successful integration in NF54 $4^{\text {luc }}$. Dd2 $2^{\text {luc }}$ is included as control. In the case of Dd2 $2^{\text {luc }}$, no product is expected across the cg6-attB locus as this is disrupted with the hdhfr drug selection cassette. (C) Stage-specific northern blot using probes to Pfpcna and luc transcripts. The expected sizes of the predominant transcripts of $1.8 \mathrm{~Kb}$ and $2.6 \mathrm{~Kb}$, respectively, were found. T1 to T5 represent timepoints of sampling from early rings, late rings, early trophozoites, late trophozoites and schizonts, respectively. (D) Time-course of luciferase sampling of NF54 $4^{\text {luc }}$ ( $2 \%$ parasitaemia, $2 \%$ HCT) over T1-T5. The bars represent mean RLU \pm stdev $(n=5)$ using the standard luciferase substrate. 
Table 2 Comparison of $\mathrm{IC}_{50}$ derived from bioluminescence and fluorescence-based assays

\begin{tabular}{|c|c|c|c|c|c|c|}
\hline \multirow[t]{2}{*}{ Drug $^{1}$} & \multicolumn{2}{|c|}{ MSF } & \multirow{2}{*}{$\begin{array}{c}\text { Luc } \\
\text { Dd2 }{ }^{\text {attB }}\end{array}$} & \multicolumn{2}{|c|}{ MSF } & \multirow{2}{*}{$\begin{array}{c}\text { Luc } \\
\text { NF54 }\end{array}$} \\
\hline & $\mathrm{Dd} 2^{\text {attB }}$ & Dd2 ${ }^{\text {luc }}$ & & NF54 ${ }^{\text {attB }}$ & NF54 & \\
\hline Artemether & $12.0 \pm 0.5$ & $11.3 \pm 0.3$ & $8.4 \pm 0.8$ & $12.1 \pm 1.3$ & $13.1 \pm 0.9$ & $12.2 \pm 0.9$ \\
\hline Atovaquone & $1.9 \pm 0.4$ & $1.3 \pm 0.6$ & $1.7 \pm 0.1$ & $0.7 \pm 0.6$ & $0.9 \pm 0.2$ & $2.9 \pm 0.3$ \\
\hline Azithromycin & $>1580$ & $>1580$ & $>1580$ & $>1580$ & $>1580$ & $>1580$ \\
\hline Cloroquine & $22.6 \pm 2.5$ & $26.4 \pm 1.0$ & $18.6 \pm 2.5$ & $26.7 \pm 1.0$ & $13.2 \pm 2.1$ & $21.0 \pm 1.3$ \\
\hline Dihydroartemisinin & $133.0 \pm 7.8$ & $125.6 \pm 2.4$ & $166.3 \pm 7.4$ & $7.9 \pm 0.7$ & $14.1 \pm 2.2$ & $19.4 \pm 1.8$ \\
\hline Mefloquine & $4.4 \pm 0.5$ & $7.8 \pm 1.2$ & $6.0 \pm 0.4$ & $9.7 \pm 1.4$ & $7.1 \pm 1.2$ & $5.6 \pm 0.3$ \\
\hline Quinine & $173.5 \pm 13.0$ & $157.3 \pm 20.0$ & $115.4 \pm 9.1$ & $62.6 \pm 5.7$ & $69.0 \pm 3.2$ & $48.5 \pm 4.0$ \\
\hline WR99210 & $n d^{2}$ & nd & nd & nd & $0.55 \pm 0.13$ & $0.48 \pm 0.21$ \\
\hline
\end{tabular}

${ }^{1} \mathrm{IC}_{50}$ values ( $\mathrm{n} .3$ independent measurements in the triplicate) reported as mean \pm standard deviation in $\mathrm{nM},{ }^{2}$ not done.

unchanged from the MSF assays of the related luc transgenic lines.

\section{Discussion}

The utility of bioluminescence in single-dose HTS of small- to medium-sized chemical libraries has been readily demonstrated, providing evidence for the luciferase reporter assay as a robust and reliable platform for antimalarial drug discovery $[16,18,20]$. An evaluation of a range of commercially-available luminogenic substrates suitable for scale-up of this assay platform are evaluated here. This evaluations shows that key assay parameters such as the Z'-score and \% coefficient of variation are essentially the same irrespective of the luminogenic substrate selected. Whilst Steady-Glo appears to offer a significant advantage due to its relative signal stability, particularly when considering use of 384 or even 1536-microwell plates, this is simply ameliorated through the use of microinjection devices that deliver substrate immediately prior to detection of the bioluminescent signal. Thus, the standard luciferase substrate, the only commercial luminogenic substrate investigated here that is not co-formulated with lysis reagent (and thus less prone to foaming on microinjection) would perhaps represent the best choice for scale-up. Critically, however, bioluminescence would appear to offer few advantages over the current choice of Sybr-Green I fluorescence assay for single-dose HTS. The advantages offered by the higher $\mathrm{S} / \mathrm{N}$ ratio and very low background of bioluminescence, whilst extremely helpful for ready analysis of the data, are significantly outweighed by the increased cost of luminogenic substrate and the restriction of study to only those $P$. falciparum lines that are genetically modified to express luciferase.

The opportunities for bioluminescence would thus appear to lie elsewhere. One such area is the exploration of stage-specific activity of anti-malarial drug action. Currently, in addition to the late trophozoite-specific expression of luciferase reported here, P. falciparum transgenic lines that express peak luciferase levels in ring, schizont and different gametocyte stages exist $[15,17,20]$. The advantages in very high $\mathrm{S} / \mathrm{N}$ ratio reported here, however, are not as apparent in the studies that describe these other transgenic parasites. For example, whilst this study reports $\mathrm{S} / \mathrm{N}$ ratios between 15667 and 17746 using the Bright-Glo substrate, the same substrate only provided $\mathrm{S} / \mathrm{N}$ ratios up to 430 in a transgenic parasite where the luc gene is under the control of a Pfhrp2 promoter [20]. These findings may result from technical issues associated with choice of luminometer or perhaps, more likely, from a need to better understand the role of luc flanking sequences. Using matched Pfpcna $5^{\prime}$ and $3^{\prime}$ flanking sequences, a complete reconstitution of the absolute and temporal control of the endogenous gene over the luc reporter can be done [21]. This same observation is similarly true using a second gene, PF0660w, which is expressed in trophozoite-stage parasites (Hasenkamp et al. submitted). These findings would suggest that use of matched flanking sequences from genes subject to high levels of temporallylinked expression may offer the opportunity to develop a library of transgenic parasites suitable for the screening of stage-specific anti-malarial drug activity.

The high rate of luciferase turnover, however, may offer the most attractive opportunity for assay development. Inducing kill using supralethal doses of the translation inhibitor $\mathrm{CHX}$ indicates that luciferase has an approximate $1.5 \mathrm{hr}$ half-life in P. falciparum. This high rate of reporter turnover, accompanied by high quantum yield of light from strong matched regulatory sequences, may be exploited to facilitate the in vitro investigation of the immediate dynamics of drug treatment. Rate-of-kill is a critical determinant of anti-malarial drug action and ideally this should be as fast as possible [1]. Rapid rateof-kill is a specific stated attribute in the target product profile for a single-exposure radical cure drug, with the intention of rapidly reducing the parasite burden, and thus clinical symptoms of disease (www.mmv.org). Rateof-kill is typically first characterized during phase IIa 
clinical trials but an in vitro assay of rate-of-kill has been recently described [10]. As this recrudescence-based in vitro assay of rate-of-kill assay requires some 21-28 days post-drug selection to develop the data, the utility of a more rapid in vitro assay to explore the rate-of-kill of antimalarial drugs is potentially of interest in establishing priorities for anti-malarial drug development programmes.

\section{Conclusion}

This study provides proof-of-principle data supporting the application of bioluminescent assays to determine the relative rate-of-kill of four anti-malarial drugs. The relative rate-of-kill as judged from bioluminescent assays is the same as that previously reported from in vivo PRR and in vitro recrudescence rate-of-kill assays [10]. Critically, drugs that exhibit the same mode of action show similar relative rates-of-kill in this novel assay format. Importantly, these assays were performed in a 96-multiwell plate format over a six-hour window which would suggest some potential for development into a rapid in vitro rateof-kill assay more amenable for scale up.

\section{Competing interests}

The authors declare no competing interests.

\section{Authors' contributions}

$\mathrm{SH}$ carried out the molecular and drug assay studies and drafted the manuscript. AS and OD carried out the initial evaluation of bioluminescence assays and comparison to MSF assays. RR measured the IC50 values in the CQR and CQS transgenic lines. PH designed the study, carried out the rateof-kill experiments and prepared the final manuscript. All authors read and approved the final manuscript.

\section{Acknowledgements}

We would like to thank Catherine Merrick for comments provided during the preparation of this manuscript. We are grateful to the Nuffield Foundation and Wellcome Trust for vacation research bursaries provided to AS and OD, respectively. This work was funded Royal Society Research Grant to PH and SH. PH is supported by a BBSRC New Investigator Award (BB/H002405/1).

\section{Author details}

'Institute for Science and Technology in Medicine, Keele University, Staffordshire, UK. ${ }^{2}$ School of Life Sciences, Keele University, Staffordshire, UK.

${ }^{3}$ School of Medicine, Keele University, Staffordshire, ST5 5BG, UK.

Received: 27 November 2012 Accepted: 3 February 2013

Published: 8 February 2013

\section{References}

1. Burrows JN, Leroy D, Lotharius J, Waterson D: Challenges in antimalarial drug discovery. Future Med Chem 2011, 3:1401-1412.

2. Grimberg BT, Mehlotra RK: Expanding the antimalarial drug arsenal-now, but how? Pharmaceuticals (Basel) 2011, 4:681-712.

3. Gamo FJ, Sanz LM, Vidal J, de Cozar C, Alvarez E, Lavandera JL, Vanderwall DE, Green DV, Kumar V, Hasan S, Brown JR, Peishoff CE, Cardon LR, GarciaBustos JF: Thousands of chemical starting points for antimalarial lead identification. Nature 2010, 465:305-310.

4. Guiguemde WA, Shelat AA, Bouck D, Duffy S, Crowther GJ, Davis PH Smithson DC, Connelly M, Clark J, Zhu F, Jiménez-Díaz MB, Martinez MS, Wilson EB, Tripathi AK, Gut J, Sharlow ER, Bathurst I, El Mazouni F, Fowble JW, Forquer I, McGinley PL, Castro S, Angulo-Barturen I, Ferrer S, Rosenthal PJ, Derisi JL, Sullivan DJ, Lazo JS, Roos DS, Riscoe MK, et al: Chemical genetics of Plasmodium falciparum. Nature 2010, 465:311-315.

5. Plouffe D, Brinker A, McNamara C, Henson K, Kato N, Kuhen K, Nagle A, Adrian F, Matzen JT, Anderson P, Nam TG, Gray NS, Chatterjee A, Janes J,
Yan SF, Trager R, Caldwell JS, Schultz PG, Zhou Y, Winzeler EA: In silico activity profiling reveals the mechanism of action of antimalarials discovered in a high-throughput screen. Proc Natl Acad Sci USA 2008, 105:9059-9064.

6. Bahamontes-Rosa N, Rodriguez-Alejandre A, Gonzalez-del-Rio R, GarciaBustos JF, Mendoza-Losana A: A new molecular approach for cidal vs static antimalarial determination by quantifying mRNA levels. Mol Biochem Parasitol 2012, 181:171-177.

7. Lelievre J, Almela MJ, Lozano S, Miguel C, Franco V, Leroy D, Herreros E: Activity of clinically relevant antimalarial drugs on Plasmodium falciparum mature gametocytes in an ATP bioluminescence "transmission blocking" assay. PLoS One 2012, 7:e35019.

8. Angulo-Barturen I, Jimenez-Diaz MB, Mulet T, Rullas J, Herreros E, Ferrer S, Jimenez E, Mendoza A, Regadera J, Rosenthal PJ, Bathurst I, Pompliano DL, Gómez De Las Heras F, Gargallo-Viola D: A murine model of falciparum-malaria by in vivo selection of competent strains in non-myelodepleted mice engrafted with human erythrocytes. PLoS One 2008, 3:e2252.

9. Paguio MF, Bogle KL, Roepe PD: Plasmodium falciparum resistance to cytocidal versus cytostatic effects of chloroquine. Mol Biochem Parasitol 2011, 178:1-6.

10. Sanz LM, Crespo B, De-Cozar C, Ding XC, Llergo JL, Burrows JN, GarciaBustos JF, Gamo FJ: P. falciparum in vitro killing rates allow to discriminate between different antimalarial mode-of-action. PLoS One 2012, 7:e30949.

11. Desjardins RE, Canfield CJ, Haynes JD, Chulay JD: Quantitative assessment of antimalarial activity in vitro by a semiautomated microdilution technique. Antimicrob Agents Chemother 1979, 16:710-718.

12. Baniecki ML, Wirth DF, Clardy J: High-throughput Plasmodium falciparum growth assay for malaria drug discovery. Antimicrob Agents Chemother 2007, 51:716-723.

13. Bennett TN, Paguio M, Gligorijevic B, Seudieu C, Kosar AD, Davidson E, Roepe PD: Novel, rapid, and inexpensive cell-based quantification of antimalarial drug efficacy. Antimicrob Agents Chemother 2004, 48:1807-1810.

14. Smilkstein M, Sriwilaijaroen N, Kelly JX, Wilairat P, Riscoe M: Simple and inexpensive fluorescence-based technique for high-throughput antimalarial drug screening. Antimicrob Agents Chemother 2004, 48:1803-1806

15. Adjalley SH, Johnston GL, Li T, Eastman RT, Ekland EH, Eappen AG, Richman A, Sim BK, Lee MC, Hoffman SL, Fidock DA: Quantitative assessment of Plasmodium falciparum sexual development reveals potent transmissionblocking activity by methylene blue. Proc Natl Acad Sci U S A 2011, 108:E1214-E1223.

16. Che P, Cui L, Kutsch O, Cui L, Li Q: Validating a firefly luciferase-based high-throughput screening assay for antimalarial drug discovery. Assay Drug Dev Technol 2012, 10:61-68.

17. Cui L, Miao J, Wang J, Li Q, Cui L: Plasmodium falciparum: development of a transgenic line for screening antimalarials using firefly luciferase as the reporter. Exp Parasitol 2008, 120:80-87.

18. Ekland EH, Schneider J, Fidock DA: Identifying apicoplast-targeting antimalarials using high-throughput compatible approaches. FASEB J 2011, 25:3583-3593.

19. Franke-Fayard B, Djokovic D, Dooren MW, Ramesar J, Waters AP, Falade MO, Kranendonk M, Martinelli A, Cravo P, Janse CJ: Simple and sensitive antimalarial drug screening in vitro and in vivo using transgenic luciferase expressing Plasmodium berghei parasites. Int J Parasitol 2008, 38:1651-1662

20. Lucumi E, Darling C, Jo H, Napper AD, Chandramohanadas R, Fisher N, Shone AE, Jing H, Ward SA, Biagini GA, DeGrado WF, Diamond SL, Greenbaum DC: Discovery of potent small-molecule inhibitors of multidrug-resistant Plasmodium falciparum using a novel miniaturized high-throughput luciferase-based assay. Antimicrob Agents Chemother 2010, 54:3597-3604.

21. Wong EH, Hasenkamp S, Horrocks P: Analysis of the molecular mechanisms governing the stage-specific expression of a prototypical housekeeping gene during intraerythrocytic development of $P$. falciparum. J Mol Biol 2011, 408:205-221.

22. Hasenkamp S, Wong EH, Horrocks P: An improved single-step lysis protocol to measure luciferase bioluminescence in Plasmodium falciparum. Malar J 2012, 11:42. 
23. Horrocks $\mathrm{P}$, Jackson $\mathrm{M}$, Cheesman $\mathrm{S}$, White $\mathrm{JH}$, Kilbey BJ: Stage specific expression of proliferating cell nuclear antigen and DNA polymerase delta from Plasmodium falciparum. Mol Biochem Parasitol 1996, 79:177-182.

24. Lambros C, Vanderberg JP: Synchronization of Plasmodium falciparum erythrocytic stages in culture. J Parasitol 1979, 65:418-420

25. Nkrumah LJ, Muhle RA, Moura PA, Ghosh P, Hatfull GF, Jacobs WR, Fidock DA: Efficient site-specific integration in Plasmodium falciparum chromosomes mediated by mycobacteriophage Bxb1 integrase. Nat Methods 2006, 3:615-621.

26. Zhang JH, Chung TD, Oldenburg KR: A simple statistical parameter for use in evaluation and validation of high throughput screening assays. J Biomol Screen 1999, 4:67-73.

doi:10.1186/1475-2875-12-58

Cite this article as: Hasenkamp et al:: Evaluation of bioluminescencebased assays of anti-malarial drug activity. Malaria Journal 2013 12:58

\section{Submit your next manuscript to BioMed Central and take full advantage of:}

- Convenient online submission

- Thorough peer review

- No space constraints or color figure charges

- Immediate publication on acceptance

- Inclusion in PubMed, CAS, Scopus and Google Scholar

- Research which is freely available for redistribution 